\title{
Paraphimosis in a 27-year-old male after sexual intercourse - non-surgical approach useful for physicians
}

Authors: Karolina Kulakowska ${ }^{1}$

${ }^{1}$ Department of Anatomical, Histological, Forensic and Locomotor System Sciences, Sapienza University of Rome, Rome, Italy

DOI: https://doi.org/10.26800/LV-142-supp5-11

\section{Background:}

Paraphimosis is a rare medical condition, but a common urologic emergency that can be presented to physicians of many backgrounds. Prompt diagnosis and treatment are important, hence it should be useful for any clinician to be familiar with its proper management. Many clinic-based, non-surgical treatments are available and preventive efforts exist in order to help patients who are at high risk of the condition. The case report presents an unusual case of a healthy young adult male with paraphimosis after sexual intercourse.

\section{Case Presentation:}

A 27-year-old male presented with painful penile swelling and inability to reduce his foreskin for two days. Following intercourse, he fell asleep without replacing foreskin and was unable to return it to its usual location next morning. After experiencing increasing pain and swelling he went to the Emergency Room. During anamnesis he denied experiencing previous paraphimoses. No piercings, obstructive symptoms, sexually transmitted diseases, urinary tract infections were reported. On physical examination, evident edema of the foreskin was observed, forming a tight collar around the glans penis, the latter appearing red. Without any evidence of sexually transmitted diseases nor dermatological issues, an urgent urologic consultation was provided. To reduce edema, the urologist squeezed the glans for some minutes, successfully allowing reduction of foreskin. The patient was later advised to always replace the foreskin.

\section{Conclusion:}

Even though an urgent consultation with a urologist is advised, many non-surgical options exist. To facilitate edema reduction common techniques include squeezing the glans, injecting hyaluronidase, applying ice. More invasive options include a "puncture" technique and direct blood aspiration. Preventive measures include daily cleaning and reducing retracted foreskin. Physicians should be aware of preventive measures and treatment of paraphimosis. Considering there are multiple nonsurgical techniques, simple and fast treatment can be applied.

Keywords: Paraphimosis, Urologic Emergency, Urology 